\title{
A poroelastic fluid/structure-interaction model of cerebrospinal fluid dynamics in the cord with syringomyelia and adjacent subarachnoid-space stenosis DOI:
}

10.1115/1.4034657

\section{Document Version}

Accepted author manuscript

Link to publication record in Manchester Research Explorer

Citation for published version (APA):

Bertram, C. D., \& Heil, M. (2016). A poroelastic fluid/structure-interaction model of cerebrospinal fluid dynamics in the cord with syringomyelia and adjacent subarachnoid-space stenosis. Journal of Biomechanical Engineering, 139(1), [011001]. https://doi.org/10.1115/1.4034657

\section{Published in:}

Journal of Biomechanical Engineering

\section{Citing this paper}

Please note that where the full-text provided on Manchester Research Explorer is the Author Accepted Manuscript or Proof version this may differ from the final Published version. If citing, it is advised that you check and use the publisher's definitive version.

\section{General rights}

Copyright and moral rights for the publications made accessible in the Research Explorer are retained by the authors and/or other copyright owners and it is a condition of accessing publications that users recognise and abide by the legal requirements associated with these rights.

\section{Takedown policy}

If you believe that this document breaches copyright please refer to the University of Manchester's Takedown Procedures [http://man.ac.uk/04Y6Bo] or contact uml.scholarlycommunications@manchester.ac.uk providing relevant details, so we can investigate your claim.

\section{OPEN ACCESS}




\title{
A poroelastic fluid/structure-interaction model of cerebrospinal fluid dynamics in the cord with syringomyelia and adjacent subarachnoid-space stenosis
}

\author{
C.D. Bertram ${ }^{1}$ and M. Heil ${ }^{2}$ \\ ${ }^{1}$ School of Mathematics \& Statistics, University of Sydney, New South Wales, Australia 2006 \\ ${ }^{2}$ School of Mathematics, University of Manchester, Manchester, United Kingdom M13 9PL
}

\begin{abstract}
An existing axisymmetric fluid/structure-interaction model of the spinal cord, pia mater, subarachnoid space and dura mater in the presence of syringomyelia and subarachnoid space stenosis was modified to include porous solids. This allowed investigation of a hypothesis for syrinx fluid ingress from cerebrospinal fluid. Gross model deformation was unchanged by the addition of porosity, but pressure oscillated more in the syrinx and the subarachnoid space below the stenosis. The poroelastic model still exhibited elevated mean pressure in the subarachnoid space below the stenosis, and in the syrinx. With realistic cord permeability, there was slight oscillatory shunt flow bypassing the stenosis via the porous tissue over the syrinx. Weak steady streaming flow occurred in a circuit involving craniocaudal flow through the stenosis and back via the syrinx. Mean syrinx volume was scarcely altered when the adjacent stenosis bisected the syrinx, but increased slightly when the syrinx was predominantly located caudal to the stenosis. The fluid content of the tissues over the syrinx oscillated, absorbing most of the radial flow seeping from the subarachnoid space so that it did not reach the syrinx. To a lesser extent, this cyclic swelling in a boundary layer of cord tissue just below the pia occurred all along the cord, representing a mechanism for exchange of interstitial fluid and cerebrospinal fluid which could explain recent tracer findings without invoking perivascular conduits. The model demonstrates that syrinx volume increase is possible when there is subarachnoid space stenosis and the cord and pia are permeable.
\end{abstract}

\section{Introduction}

Syringomyelia is a neurological disease condition characterised by the existence of one or more macroscopic fluid-filled cavities (syrinxes) in the spinal cord. The resulting hindrance to normal cord neurotransmission can lead to pain, loss of sensation, loss of motor control, and other neurological deficits. Syringomyelia usually develops either in association with congenital Chiari I malformation or as a late consequence of traumatic spinal injury, the common element being some degree of obstruction to the free flow of cerebrospinal fluid (CSF) in the spinal subarachnoid space (SSS). The condition is treated surgically, by draining the syrinx(es) or relieving the obstruction or both, but the long-term outcome is typically poor even if short-term symptomatic relief is obtained [22]. 
Hydrodynamics is conjectured to play a major role in the genesis of a syrinx, and accordingly there have been various attempts to model the physics of the situation, as reviewed by Elliott et al. [13]. Bertram [2] developed an axisymmetric fluid/structure-interaction (FSI) model of the whole tapered spinal cord, SSS and dura mater, with excitation consisting of pressure transients or waveforms applied either to the cranial end of the SSS or to the outside of the abdominal dura. A substantial syrinx with dimensions and approximate location (spinal segments C4 to T3) as shown in fig. 1 of [1] was included, as were representations of the filum terminale and the pia mater. The SSS was partially occluded by a trapezoidal obstruction of maximum length $30 \mathrm{~mm}$, extending from the dura and occupying up to $90 \%$ of the SSS cross-section by area. All solids were assumed linearly viscoelastic; the CSF and syrinx fluid were assigned the properties of water. The SSS stenosis was located halfway along that part of the cord occupied by the syrinx.

Sinusoidal pressure at $2.5 \mathrm{~Hz}$ at the cranial end of the SSS gave rise to a to-and-fro surging motion of the CSF into the blind-ended SSS, only somewhat lessened by the stenosis, across which there was a large pressure drop when flow past the stenosis was maximal. In turn this caused the syrinx to experience (through the pia and thinned overlying cord) an oscillating SSS pressure effectively divided into two halves. The syrinx deflected accordingly, with its contents also surging from end to end to an extent agreeing with MRI measurement [7]. This 'slosh' was identified as a potential cause of tearing stress at the syrinx ends that could lead to syrinx lengthening, confirming a hypothesis by Williams [33]. When pressure was averaged over the $2.5 \mathrm{~Hz}$ cycle, that in the SSS was elevated caudal to the stenosis, which acted as a very leaky one-way valve (confirming a phenomenon first discovered in a physical model [23]). The average pressure in the syrinx was also elevated relative to that in the SSS cranial to the stenosis (and relative to its initial value), but only about one-third as much.

Since the solids were all impermeable, and the syrinx fluid was effectively incompressible, the syrinx volume was fixed in this model. But on the basis of the above average-pressure findings, the hypothesis was generated that if the pia and thinned cord had been uniformly porous over the length of the syrinx, the

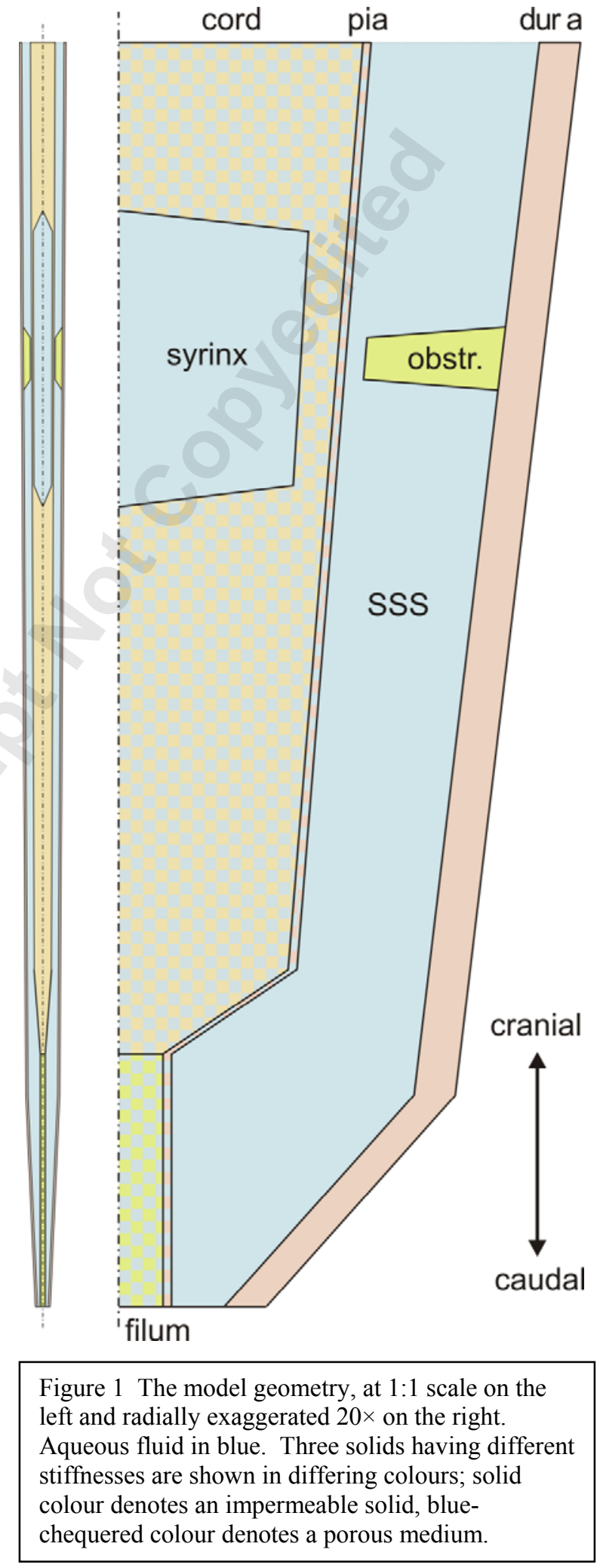


radial pressure difference caudal to the stenosis would drive fluid into the syrinx, while the smaller corresponding cranial difference would drain syrinx fluid at a lesser rate. Thus on balance these flows would cause syrinx volume to increase, representing a possible mechanism of syrinx enlargement and syrinx filling with CSF.

In this work we test this hypothesis, by converting the previous model to one in which all the relevant solids are poroelastic, allowing the possibility of fluid seepage between the SSS and the syrinx, and of variation in syrinx volume. We also conduct a mathematical analysis in a simplified geometry. It transpires that the behaviour of the poroelastic media is rather more complex than was naïvely assumed at the outset, being dominated, insofar as cyclic events are concerned, by changes in the fluid content. Our findings have significance beyond the confines of syringomyelia, as well as illuminating the combined potential role of permeable solids and SSS stenosis in syrinx filling.

\section{Methods}

We here present an outline of our methods; a more detailed exposé is given elsewhere [15]. The geometry of the model is shown in Figure 1, in two views. On the left, a cross-section of the whole axisymmetric model is shown with equal axial and radial scaling, emphasizing its slenderness. The maximum radial dimension, over the dura at the cranial end, is $11 \mathrm{~mm}$, while the total length to the sacral end of the filum terminale, SSS and dura is $600 \mathrm{~mm}$. These and all other dimensions are unchanged from our impermeable model [2]. In the view on the right, only a half cross-section of the model is shown, with the axis of symmetry on the left (dot-dash line), and the radial scale is increased $20 \times$ relative to the axial scale, allowing ready identification of (1) the poroelastic cord tapering down to the poroelastic filum at the sacrum, (2) the contained syrinx filled with aqueous fluid, (3) the cordbounding pia, of uniform thickness $0.2 \mathrm{~mm}$, (4) the SSS filled with aqueous CSF, (5) the dura bounding the SSS at its outer margin, and (6) the localised SSS obstruction forming the stenosis. As in the impermeable model, the dura is not an exact representation of the real dura, but its geometric and elastic properties were together chosen to yield a reasonable emulation of the in vivo stiffness presented by the dura as backed by fat and bone (see [4] for more detail). The stenosis here corresponded to the most severe of the previous three [2], occluding $87.5 \%$ of the SSS locally by radius and $90.3 \%$ by area.

Following Simon [30], the poroelastic model is governed by the equations of momentum conservation for the poroelastic medium

$$
\widetilde{\nabla} \cdot \overline{\bar{\sigma}}=\rho_{\mathrm{pe}} \frac{\partial^{2} \tilde{u}}{\partial t^{2}}+\rho_{\mathrm{f}} \frac{\partial \tilde{q}}{\partial t}
$$

and for the pore fluid

$$
-\widetilde{\nabla} p_{\mathrm{p}}-\frac{\mu}{k} \tilde{q}=\rho_{\mathrm{f}} \frac{\partial^{2} \tilde{u}}{\partial t^{2}}+\frac{\rho_{\mathrm{f}}}{\varphi} \frac{\partial \tilde{q}}{\partial t}
$$

together with the constitutive equation for the poroelastic medium

$$
\overline{\bar{\sigma}}+\alpha p_{\mathrm{p}} \overline{\bar{I}}=\frac{E v}{(1+v)(1-2 v)}(\widetilde{\nabla} \cdot \tilde{u}) \overline{\bar{I}}+\frac{E}{1+v} \overline{\bar{e}}
$$

and the mass conservation equation for the pore fluid

$$
\alpha \widetilde{\nabla} \cdot \frac{\partial \tilde{u}}{\partial t}+\widetilde{\nabla} \cdot \tilde{q}=0
$$


where $\overline{\bar{\sigma}}=$ poroelastic matrix stress, $\tilde{u}=$ matrix displacement, $\tilde{q}=$ seepage velocity (relative to the matrix), $p_{\mathrm{p}}=$ pore fluid pressure, $\overline{\bar{e}}=$ matrix strain and $t=$ time. The matrix strain is a function of the matrix displacement,

$$
\overline{\bar{e}}=\frac{1}{2}\left(\widetilde{\nabla} \tilde{u}+(\widetilde{\nabla} \tilde{u})^{\mathrm{T}}\right)
$$

The constants are the density $\rho_{\mathrm{pe}}$ of the matrix and pore fluid combined, the fluid density $\rho_{\mathrm{f}}$ and viscosity $\mu$, the matrix permeability $k$ and porosity $\varphi$, the Biot-Willis parameter $\alpha$, the elastic modulus $E$ and the (drained) Poisson ratio $v$. In addition, the usual Navier-Stokes equations were solved for the fluid in the SSS and in the syrinx. Where a solid was required to be impermeable, $\tilde{q}$ and $p_{\mathrm{p}}$ were set to zero and equations 2 and 4 were not used. Fluid/structure interaction was accomplished by enforcing at the boundaries between the fluid (blue in Fig. 1) and poroelastic (chequered in Fig. 1) domains that (i) the pore fluid pressure equalled the normal component of stress in the fluid domain (arising from pressure and viscous stress),

(ii) the traction acting on the matrix equalled that exerted by the fluid,

(iii) the normal component of matrix velocity plus the pore fluid seepage velocity equalled the normal component of velocity in the fluid domain, and

(iv) the tangential component of matrix velocity equalled that in the fluid domain ${ }^{1}$.

At all other matrix boundaries, traction or displacement was prescribed. At the pore fluid boundaries either the pore pressure or the normal component of velocity was imposed. On the remaining boundaries of the fluid domains, traction or velocity was specified; thus syrinx fluid could not cross the axis of symmetry, and CSF encountered a wall at the sacral end of the SSS. The excitation was applied as a time-varying normal traction at the cervical open boundary of the SSS, to mimic the CSF pulse pressure arising from cranial arterial pulsatility. Axial motion of the solids was prevented at both ends of the model.

For the sake of comparison, we sought as far as possible to emulate the parameter values used with the impermeable model [2]. Thus the amplitude of the pressure forcing applied to the cranial SSS was again $500 \mathrm{~Pa}$, and frequency $2.5 \mathrm{~Hz}$. The elastic stiffness of the solids was unchanged from before [2], at $5 \mathrm{kPa}$ for the cord [26], $62.5 \mathrm{kPa}$ for the filum and the block obstructing the SSS, and 1.25 MPa for the pia and dura. However viscoelastic properties were here omitted, since (unpublished) comparison of results for viscoelastic and purely elastic solids in the former impermeable model showed negligible difference when excitation was by $2.5 \mathrm{~Hz}$ sine waves ${ }^{2}$. All fluid and poroelastic-media densities were set to $10^{3} \mathrm{~kg} \mathrm{~m}^{-3}$, and the fluid viscosity was set to $10^{-3} \mathrm{~Pa} \mathrm{~s}$. The drained Poisson ratio of the poroelastic material matrices (cord, pia and filum) was set to 0.35 [31], and their porosity to 0.3 ; the Poisson ratio of the impermeable solids was set to 0.49 . As befits a fully liquid-saturated poroelastic medium, the value of the Biot-Willis parameter was set to 1 .

The pia mater has been reported anatomically to be variably fenestrated along the length of the spinal cord [9], but its permeability to fluid seepage has not been measured to our knowledge. In the absence of better information, we took the pia and the cord to have the same (isotropic) permeability, set to either $10^{-12}, 10^{-13}$ or $10^{-14} \mathrm{~m}^{2}$, of which the last is believed to be the most realistic [31].

\footnotetext{
${ }^{1}$ This last condition enforces no slip velocity at the poroelastic interface, i.e. amounts to setting the Beavers-JosephSaffman inverse slip rate coefficient [19] to zero.

${ }^{2}$ As distinct from isolated $5 \mathrm{~ms}$ transients, which set up waves that decay at a rate set by the solid viscoelasticity and the fluid viscosity — see [2] and [3]. Such transients were not used here.
} 
The above permeable model was compared with a recreation in new code of the former impermeable model [2]; here the Poisson ratio of all solids was set as before to 0.49 . We also found alternative versions of the permeable model useful in reaching a comprehensive understanding of the mechanics. Thus we also derived results from a version in which the Biot-Willis parameter was set to 0 ; this deprives the fluid seepage of the opportunity to interact with the poroelastic matrix so as to deform it in any way, while retaining the pressure-driven Darcy flow through the porous medium. We also examined a version of the model in which only that part of the cord and pia overlying the main quasicylindrical part of the syrinx was poroelastic, with the rest of the structure impermeable.

The coupled Navier-Stokes/poroelastic model was realized in the open-source finite-element software oomph-lib [16], available at www.oomph-lib.org. Implementing the equations of poroelasticity in this software involved new programming, and exhaustive tests were run to validate the new code; see [15]. Results from the poroelastic model with parameters set for no permeability were also compared in detail with results from the former implementation of the impermeable fluid/structure-interaction model in the commercial finite-element code ADINA (ADINA R\&D Inc., Watertown MA, USA). However, whereas that implementation used block-structured grids to divide the fluid and solid parts of the model into finite elements, all grids used here were unstructured. Grid refinement testing was performed to ensure that the results reported here are independent of grid spacing, and that all fluid flows were properly resolved. The same time-step $(5 \mathrm{~ms})$ was used as before [2], and we also tested to ensure that this time-step was adequate.

\section{Results}

\subsection{Displacement and pressure}

The overall axial and radial deformations of the model in response to the sinusoidal forcing were almost entirely unaltered by the incorporation of porosity; see Figure 2. Note that the impermeable-
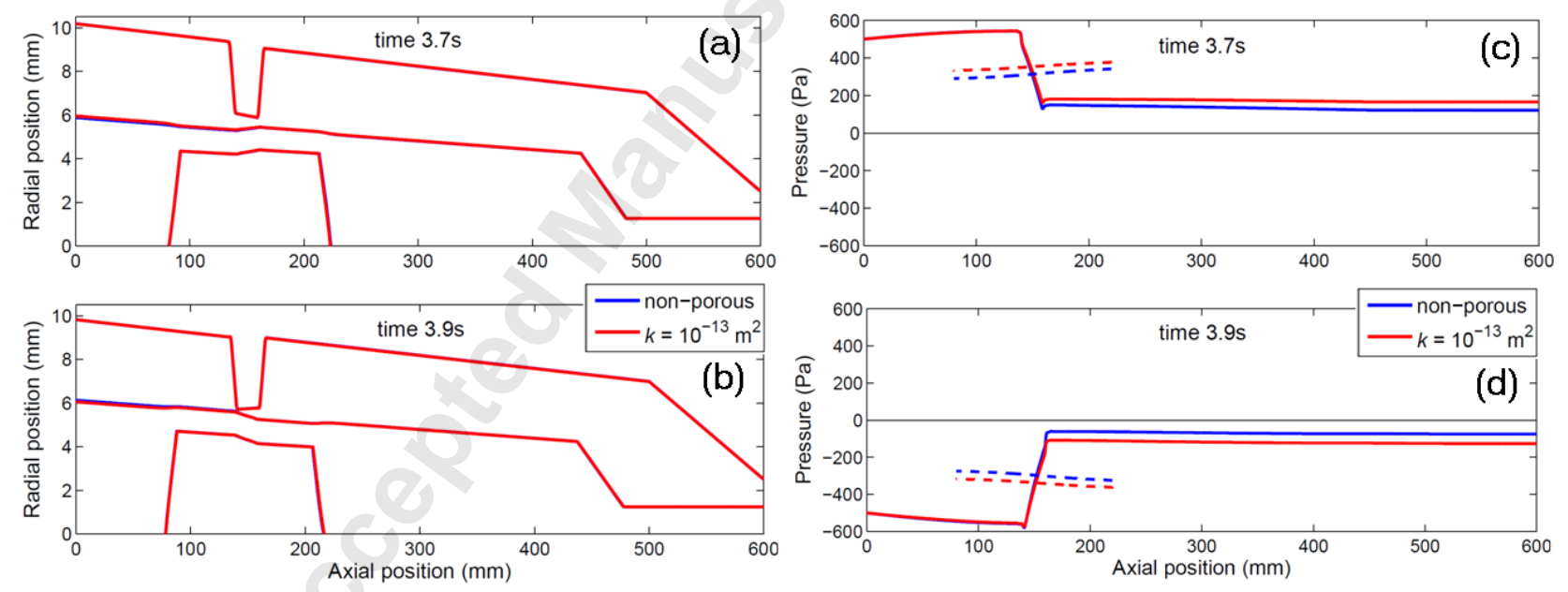

Figure 2 The deflection ( $a, b)$ of the FSI boundaries of the model, magnified $5 \times$, and the pressure along the inner boundary of the SSS (solid line) and (broken line) on the syrinx centre-line (c, d), both shown at the peak (a, c) and the trough $(b, d)$ of the forcing cycle. The permeable model outline (red) almost entirely overlaps that of the impermeable model (blue). The real change in gap width is much smaller than depicted in the exaggerated views (a, b) shown here.

model deformations differ from those shown in fig. 8 of [2] because (i) the deformation magnification is smaller here, (ii) the excitation sine-wave here has zero mean, and (iii) radial motion at the cervical extremity of the model is here permitted. The deformation of all parts of the structure causes the 
stenosis gap to be larger when the excitation pressure is high, pushing SSS flow craniocaudally, than when it is low, allowing caudocranial flow. However, the actual change in gap width was much smaller than as depicted in Fig. 2; for $k=10^{-13} \mathrm{~m}^{2}$, the minimum gap width varied by $\pm 9.3 \%$ about a mean of $0.440 \mathrm{~mm}$ over a cycle of excitation. There are also non-negligible axial displacements of the cord between the two extremities of the model where it is fixed axially, causing the whole syrinx to move caudally in panel a and cranially in panel b. Also shown in Fig. 2 is the pressure in the SSS and in the syrinx at the same two instants of the forcing cycle. The pressure at axial coordinate $z=0$ is the value imposed by the excitation. As $z$ increases, the amplitude of the pressure variation during the cycle increases up to the start of the stenosis. A large pressure drop occurs across the stenosis, corresponding to caudally directed flow in panel $\mathrm{c}$ and cranially directed flow in panel $\mathrm{d}$. Consequently the amplitude of the SSS pressure variation caudal to the stenosis is greatly reduced. The instantaneous pressure in the syrinx stays about halfway between the SSS pressures on either side of the stenosis, with a gradient accelerating the intra-syrinx flow (which is inertially dominated - see fig. 10 of [2]) in the opposite direction to SSS flow through the stenosis gap (which is viscously dominated) at the two instants depicted. When the whole cord and pia are permeable, the amplitude of the pressure variation in the SSS caudal to the stenosis and in the syrinx is significantly greater than when they are impermeable.

From Fig. 2(c) and (d), it can be seen that the SSS pressure swing in the blind-ended region caudal to the stenosis is not symmetrical about zero. This is confirmed by the average over a cycle, as shown in Figure 3, which is elevated in this region. Cranial to the stenosis $(z<135 \mathrm{~mm})$, the mean pressure remains at zero, the mean of the excitation applied at $z=0$. Across the stenosis itself $(135<z<165$ $\mathrm{mm})$, the mean pressure ${ }^{3}$, instead of ramping up from zero to the elevated value beyond $z=165 \mathrm{~mm}$, is depressed relative to that imagined ramp; the depression is caused by a combination of two nonlinearities: (i) a Bernoulli effect (conversion of pressure into kinetic energy) in the high-averagevelocity stenosis gap, as noted previously [2], and (ii) the nonlinearly increasing viscous resistance to flow through the gap with decreasing gap width. Because of the tapering model geometry, and also because of the annular geometry in general, the mean pressure profiles at the inner and outer edges of the gap differ.

Although the amplitude of the SSS pressure

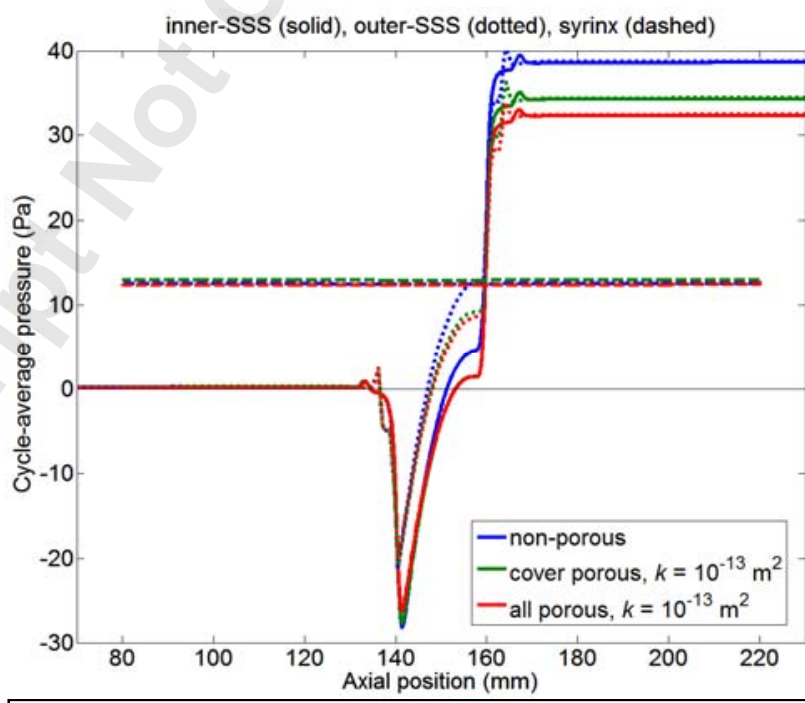

Figure 3 Profiles of cycle-average pressure vs. axial position at the inner (solid lines) and outer (dotted) edges of the SSS, and at the syrinx centre-line (dashed). Only the axial region $70<z<230 \mathrm{~mm}$, which spans the syrinx, is shown. Results are shown for the non-porous cord and pia (blue), with cord and pia porous only over the syrinx (green, "cover porous"), and with the whole cord and pia porous (red, "all porous"). variation caudal to the stenosis is greater when the cord is permeable, its mean over time is slightly less elevated than when the cord is impermeable. When the cord and pia are porous only over the syrinx,

\footnotetext{
${ }^{3}$ Note that the apparent irregularities in the mean-pressure curves at the entrance and exit of the stenosis are real, being the result of transient flow separation and eddying downstream of the stenosis (in and beyond the widening region where the trapezoidal block forming the stenosis linearly tapers back into the SSS outer wall). These instantaneous flow features were fully resolved in the Navier-Stokes equation solutions, and their effect on pressure, being nonlinear, does not average to zero over a cycle.
} 
the pressure elevation is still reduced relative to the impermeable-cord situation, but less so than when the whole cord and pia are porous. These differences in caudal SSS pressure are not reflected in the syrinx, where the cycle-average pressure is almost exactly the same under all three porosity conditions. Thus the mean syrinx pressure elevation, relative to the initial value of zero, is a larger fraction of the caudal SSS pressure elevation when the cord is porous than when it is non-porous. 


\subsection{Oscillatory flows}

A clue as to the reasons for these changes in pressure elevation can be gained by examining the oscillatory flow through the 'leaky valve' of the flexible stenosis. Once the cord and pia are porous, the flow past the stenosis is not entirely confined to the cyclically varying free gap under the block creating the stenosis; there is also the possibility of flow in parallel via the syrinx, involving transit twice through the porous media overlying the syrinx. To quantify the relative magnitude of this shunt flow, it was convenient to examine the situation where the cord and pia were porous only over the syrinx, because then the shunt flow could be assessed by integrating the velocity over one readily defined surface; see Figure 4. As shown schematically in the upper left panel, gap flow (blue arrow) was computed by integrating fluid
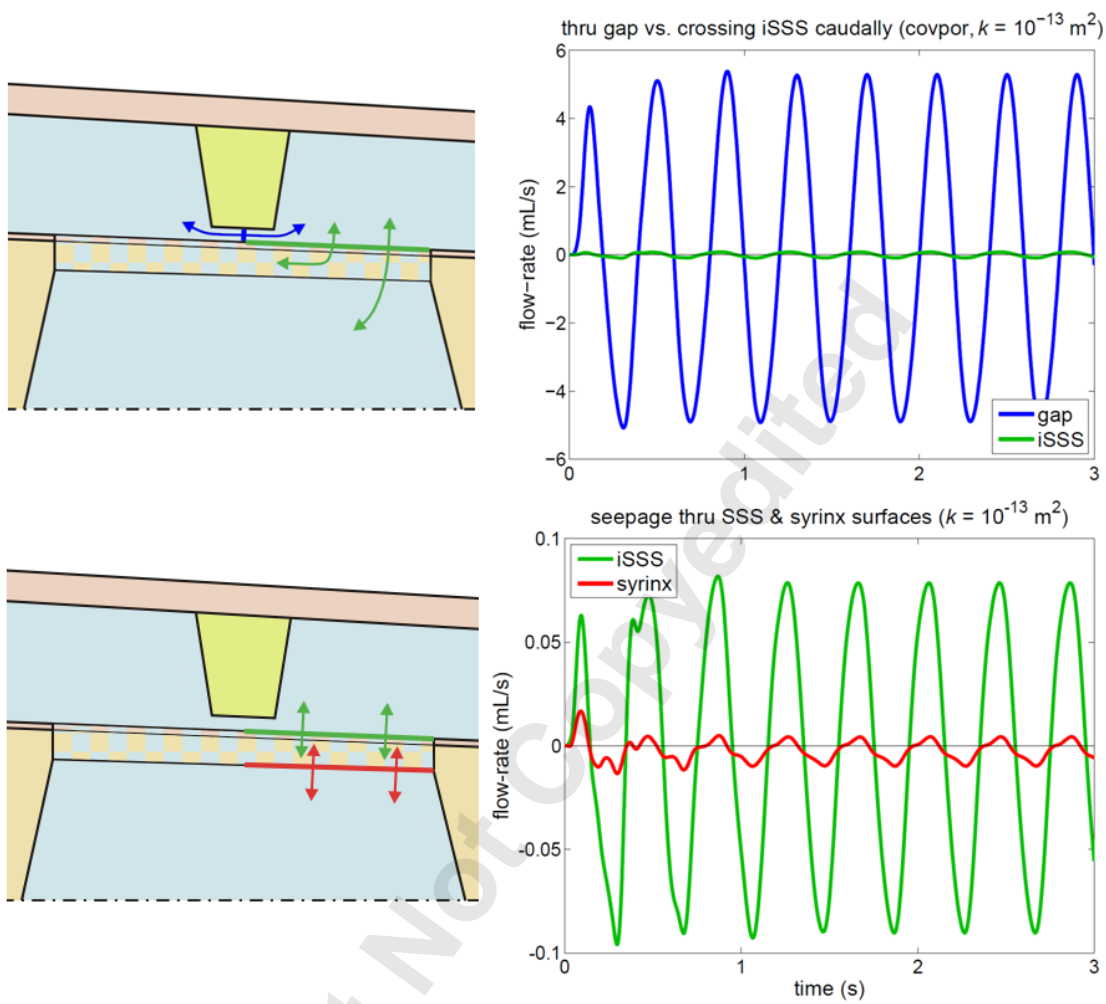

Figure 4 Upper panels compare the flow-rate through the stenosis gap (blue, "thru gap") with that arriving in the caudal SSS via the syrinx and overlying porous media (green, "crossing iSSS caudally"-iSSS denotes the inner boundary of the SSS). Lower panels compare the same flow-rate entering the caudal SSS (green) with that leaving the caudal half of the syrinx (red). Start-up transients are visible over the first three cycles (1.2 s). velocity over a surface which amounted to a radial cut (shown in blue) at the midpoint of the gap. Similarly, shunt flow into the caudal SSS was computed by integrating the seepage velocity through the $\mathrm{SSS} /$ pia interface from the stenosis midpoint to the caudal end of the porous media overlying the syrinx (green). This accounts for both flow which has shunted the stenosis gap but remained within the porous media, and that which has crossed the porous media and arrived via the syrinx (green arrows). If the whole cord and pia were porous, there would be a third component to consider, arriving in the caudal SSS from the syrinx via the cord and pia caudal to the syrinx. The calculation is still slightly approximate, because the axial location where cranial seepage into the SSS/pia interface (at the appropriate time during the cycle) becomes caudal seepage out of the interface moves during the cycle and does not conform precisely to the stenosis midpoint. As seen from the resulting waveforms (upper right), with $k=10^{-13} \mathrm{~m}^{2}$ the oscillatory shunt flow was only a small fraction (specifically, $1.66 \%$ peakto-peak for the last cycle shown) of the gap flow. Whereas the gap flow amplitude was essentially independent of the permeability $k$, the shunt flow amplitude increased with $k$ over the range from $10^{-14}$ to $10^{-12} \mathrm{~m}^{2}$ approximately as $k^{0.65}$.

Of the oscillatory flow which crossed the caudal half of the SSS/pia interface, only a small fraction emerged on the other side of the porous media overlying the syrinx, as shown in the lower panels of 
Fig. 4. The rest was absorbed ${ }^{4}$ in cyclic swelling of the cord material overlying the syrinx, i.e. the fluid content of the poroelastic medium increased and decreased, with concomitant slight oscillatory changes in the radial thickness of the overlying media. This cyclic swelling phenomenon will be examined in more detail below.

Insofar as the oscillatory events depicted in Fig. 4 are concerned, an exactly similar scenario takes place in the cranial half of the syrinx and overlying tissues, with the only change being a $180^{\circ}$ phase shift, i.e. flow into the cranial half of the syrinx occurs at the same time as flow out of the caudal half, and vice versa. However, close examination of the waveforms on the lower right of Fig. 4 reveals that both oscillations occur around a small negative mean value, representing flow from the caudal SSS into the syrinx. The corresponding mean for the cranial side is positive, denoting flow from the syrinx into the SSS cranial to the stenosis. These are two manifestations of an overall steady streaming flow in a circuit, from the cranial SSS via the gap to the caudal SSS, through the caudal half of the porous media overlying the syrinx and thus into the syrinx, and back again via the cranial half of the overlying media, as depicted in Fig. 5. The driving force for this circular flow is the elevated pressure in the caudal SSS, as created by the leaky valve effect of the flexible stenosis, but obviously it also depends on the existence of cord and pia porosity.

\subsection{Syrinx volume change}

As was shown in Fig. 3, the mean pressure in the syrinx barely changes at all with the inclusion of cord and pia permeability. The syrinx is surrounded by elastic tissue, and its original dimensions correspond to zero restoring force in those tissues.

Porous-media-induced changes in time-average transmural pressure between the SSS and the syrinx (again see Fig. 3) are tiny compared to the elastic moduli of the intervening tissues. Thus we suspect already that changes in
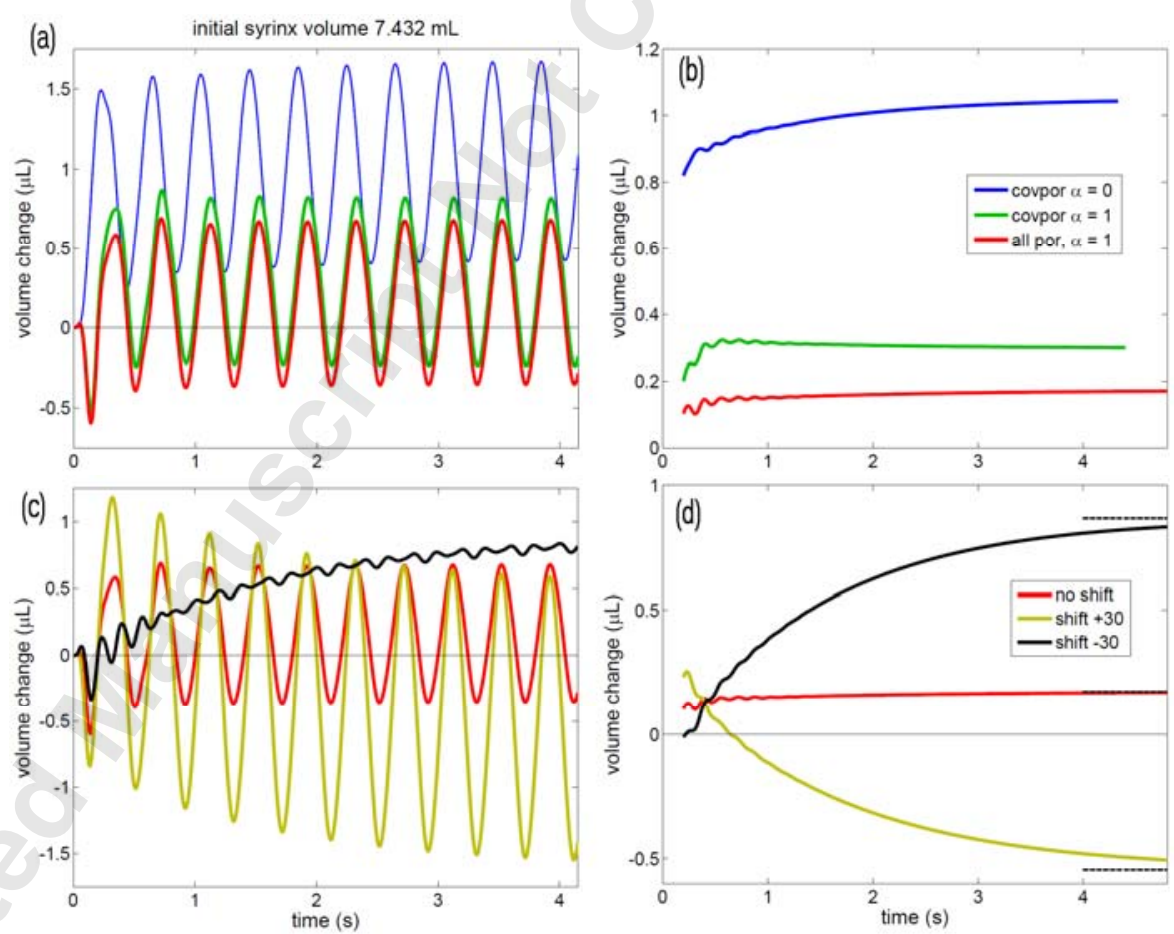

Figure $6(\mathrm{a}, \mathrm{c})$ Instantaneous changes in syrinx volume at $k=10^{-13} \mathrm{~m}^{2}$, with $(\mathrm{b}$, d) the corresponding running averages over a cycle. Panels (a) and (b) compare (red) the result when the whole cord and pia are fully poroelastic $(\alpha=1)$ to (green) that when the cord and pia are poroelastic only over the syrinx, and (blue) Darcy flow only occurs over the syrinx. Panels (c) and (d) compare (red) the same whole-cord-poroelastic result with that when the syrinx is displaced axially ("shift") by (beige) $+30 \mathrm{~mm}$ or (black) $-30 \mathrm{~mm}$ relative to the stenosis. Monoexponentials are fitted to the curves in (d) and extrapolated to find the asymptotic values (dashed lines).

\footnotetext{
${ }^{4}$ The diagram at lower left of Fig. 4 suggests another alternative, that some of the pore fluid arriving via the green interface might then seep into the cranial half of the cord and pia overlying the syrinx. However, it should be remembered that the true geometry was very slender, such that in practice this path was not significant.
} 
mean syrinx volume as a result of cord and pia porosity will be small. This is corroborated by Figure 6(a), which shows instantaneous syrinx volume changes at $k=10^{-13} \mathrm{~m}^{2}$ in response to the cyclic forcing. The volume changes are seen to take several cycles to settle down to a final steady oscillation about a constant mean. The length of this evolution (to be distinguished from short-lived start-up transients as shown in Fig. 4) is highly dependent on the permeability, lasting in excess of 100 excitation cycles at $k=10^{-14} \mathrm{~m}^{2}$. The amplitude of the cyclic volume changes also depends on the permeability (waveforms omitted for the sake of brevity), approximately as $k^{0.33}$ over $10^{-14}$ to $10^{-12} \mathrm{~m}^{2}$ with the whole cord and pia porous.

Fig. 6(a) compares volume changes when the whole cord and pia are porous (red) with those when only the cord and pia overlying the syrinx are porous (green) and when (in the latter model) pore fluid interactions with the matrix are removed and only Darcy flow persists $(\alpha=0$, blue). This last situation effectively recreates the imagined scenario which gave rise to the starting hypothesis for this work. That thinking did not encompass the full complexity of the poroelastic situation, where variations in porous-medium fluid content can play a major role (Fig. 4). While under such Darcy-flow-only conditions there is indeed seen to be a post-transient increase in mean syrinx volume (by $+0.0140 \%$ ), this already tiny increase is greatly attenuated (down to $+0.0040 \%$ ) when pore-flow interactions are allowed, and yet further reduced when the whole cord and pia are porous $(+0.0023 \%)$.

\subsection{Syrinx/stenosis axial offset}

The latter situation (red) is replotted in Figure 6(c) for comparison with the results of shifting the syrinx axially by $\pm 30 \mathrm{~mm}$. When the syrinx is shifted cranially $(+30 \mathrm{~mm}$, beige), the amplitude of instantaneous volume variations increases; when it is shifted caudally $(-30 \mathrm{~mm}$, black), the amplitude decreases. These amplitude changes appear related to the amplitude of pressure change in the adjacent SSS [see Fig. 2(c, d)], but not in any simple way, as indicated by the appearance of a second harmonic (black curve). A cranial shift relative to the stenosis causes the ultimate change in mean syrinx volume to be negative, i.e. the syrinx shrinks slightly (by $-0.0070 \%$ ). A caudal shift (see Figure 7 ) increases the ultimate mean syrinx volume, by $+0.0122 \% 5$. These figures are compared with the corresponding figures for $k=10^{-12} \mathrm{~m}^{2}$ in Table 1, where it is seen that the absolute and percentage changes were rather similar at these two values, particularly with the syrinx shifted caudally. The duration of the mean volume change evolution at $k=10^{-14} \mathrm{~m}^{2}$ meant that it was impractical to compute final posttransient values for this permeability.

\begin{tabular}{|c|c|c|c|c|}
\hline syrinx posn. & \multicolumn{2}{|c|}{$k=10^{-12} \mathrm{~m}^{2}$} & \multicolumn{2}{c|}{$k=10^{-13} \mathrm{~m}^{2}$} \\
\hline & $\Delta$ (vol.), $\mu \mathrm{L}$ & $\Delta$ (vol.), $\%$ & $\Delta$ (vol.), $\mu \mathrm{L}$ & $\Delta$ (vol.), \% \\
\hline$-30 \mathrm{~mm}$ & +0.880 & +0.0124 & +0.868 & +0.0122 \\
\hline no shift & +0.221 & +0.0030 & +0.174 & +0.0023 \\
\hline$+30 \mathrm{~mm}$ & -0.461 & -0.0059 & -0.545 & -0.0070 \\
\hline
\end{tabular}

Table 1 Mean syrinx volume changes with a shift in syrinx position and with $k$. Whole cord and pia porous, with $\alpha=1$. Monoexponential curves fitted to running cycle-averages at $t \geq 1 \mathrm{~s}$ were extrapolated beyond $\sim 5 \mathrm{~s}$ to find the final $\Delta$ (volume).

\footnotetext{
${ }^{5}$ The syrinx was shifted in such a way as to preserve its length and the thickness of the cord layer overlying it; thus its volume increased slightly with cranial shift (to $7.766 \mathrm{~mL}$ ) and decreased slightly with caudal shift (to $7.105 \mathrm{~mL}$ ). Percentages are calculated relative to the altered initial volume of the shifted syrinx.
} 

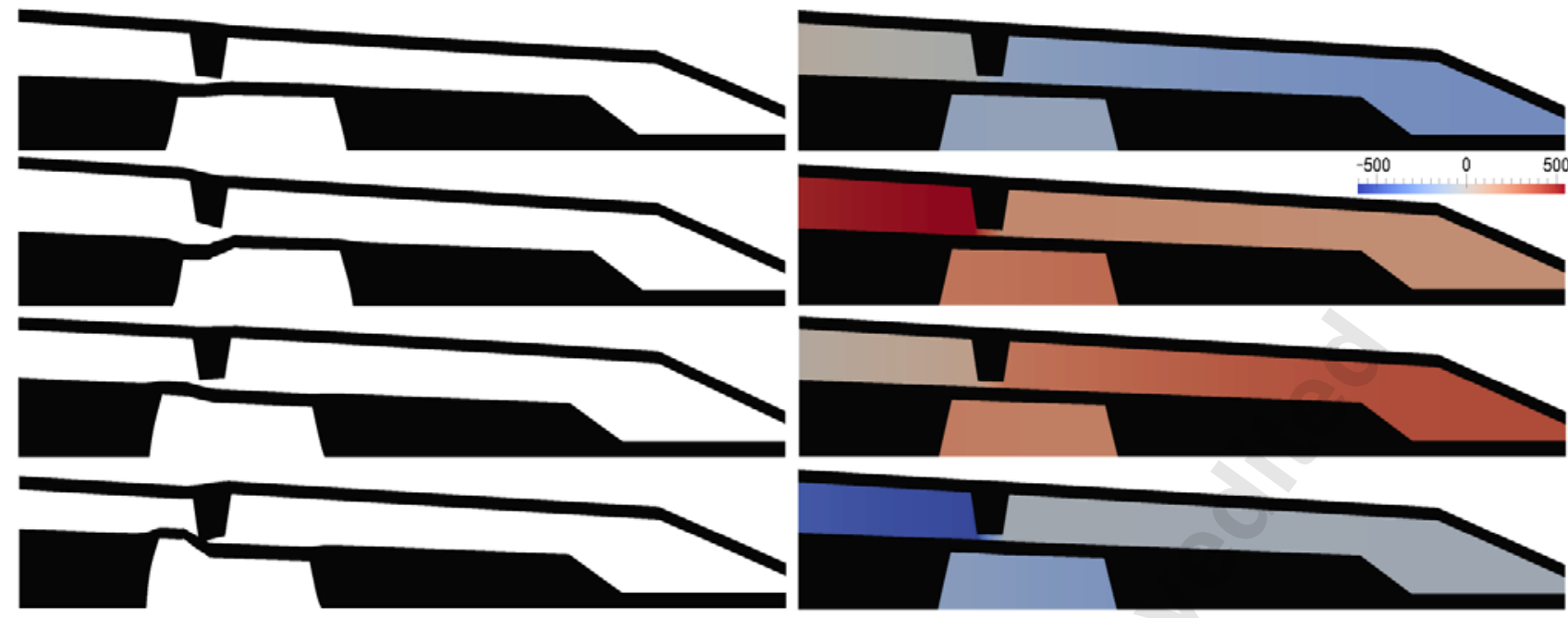

Figure 7 The solids outline and the fluid pressure at four equi-spaced times during an excitation cycle, with the syrinx displaced $30 \mathrm{~mm}$ caudally. Colour key shows pressure in Pa. Radial scale 10× axial scale, displacements exaggerated 20×. 


\subsection{Cyclic swelling}

The phenomenon of cyclic swelling, the existence of which was evident from the disparity of pulsatile seepage flow-rates into and out of the cord and pia overlying the syrinx in Fig. 4, is probed further in Figure 8. This shows profiles for a radial cut at $z=90 \mathrm{~mm}$ through the overlying media, as shown (in red) in the model sketch on the left, at eight equi-spaced times through a cycle of excitation after initial transients have decayed. The local position of the cord/pia interface, at $r=5.44 \mathrm{~mm}$, is shown in each panel by a dotted line. The upper left panel shows the radial displacement of each crosssectional location. All parts of the overlying tissues move radially in and out rather similarly, but it is noticeable that the displacements are slightly larger at the syrinx boundary on the left-hand side.
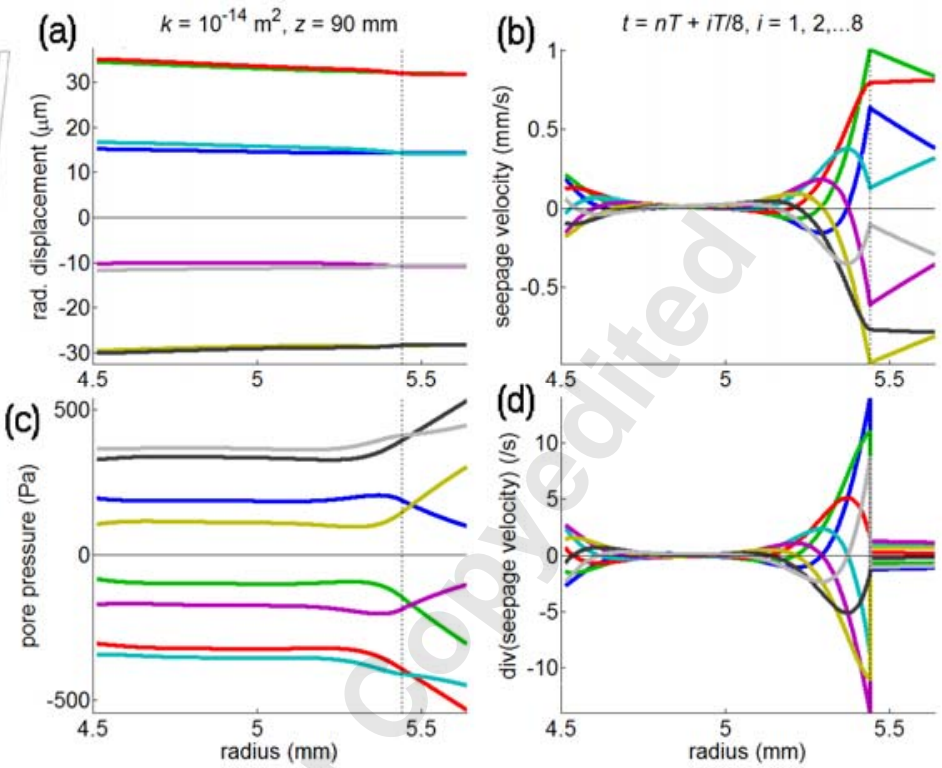

Figure 8 Instantaneous profiles of (a) radial displacement, (b) seepage velocity, (c) pore pressure, and (d) the divergence of seepage velocity, for eight equi-spaced times through the cycle, vs. radial position within a cut through the cord and pia overlying the syrinx as indicated in the sketch on the left. In each panel the syrinx is to the left, and the SSS to the right.

This is evidence of the time-varying thickness of the overlying cord, related via the Poisson ratio to the time-varying circumference of this same tissue. The seepage velocity is shown in the upper right panel. At the cord/pia interface on the right, it varies $\pm 1 \mathrm{~mm} / \mathrm{s}$. The lower right panel shows the divergence of the seepage velocity, which is the rate of change of fluid content; these profiles show explicitly the rate at which fluid is being imbibed or expelled from the porous matrix as a function of radial position and time. Because the elastic stiffness of the pia is 250 times greater than that of the cord, changes in pial fluid content are always small. The largest changes occur just inside the cord/pia interface, diminishing with depth of penetration into the cord material. A smaller but otherwise similar boundary layer occurs at the syrinx surface. Beyond its effect in limiting the volumetric strain, i.e. the swelling of the pia itself, pial stiffness has an important second effect: containment by the stiff pia greatly
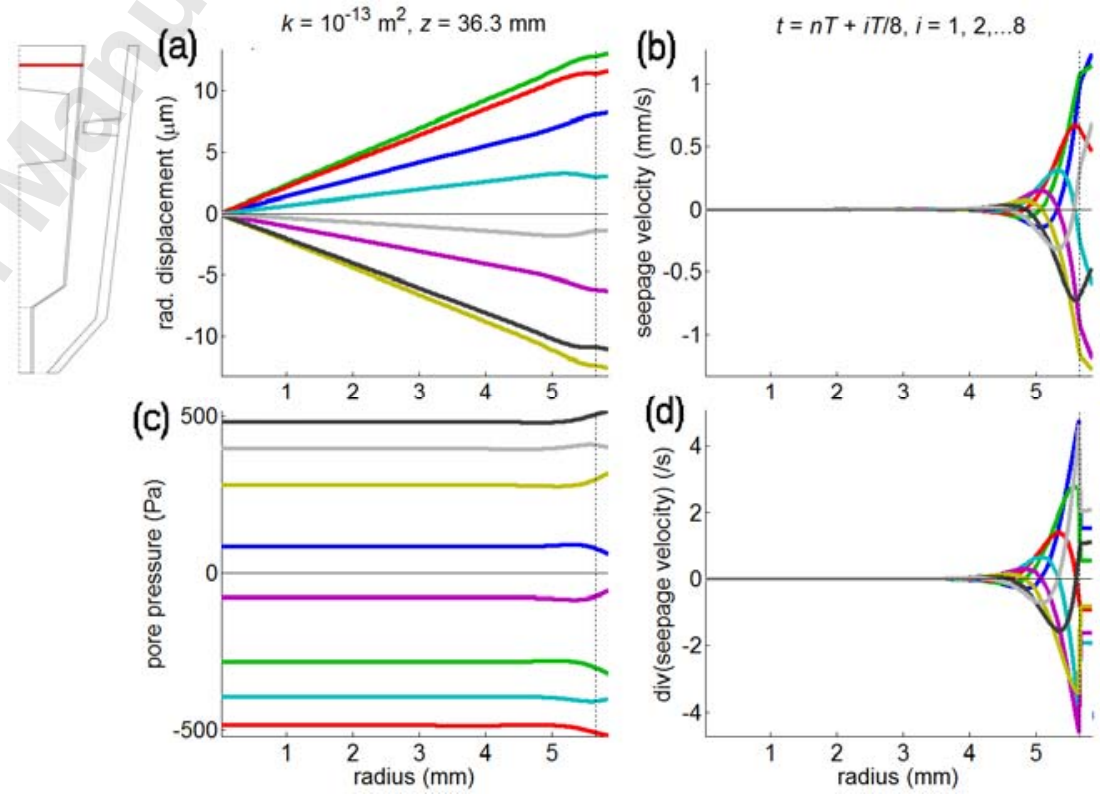

Figure 9 Traces as in Fig. 8, but for a radial cut through the cord cranial to the syrinx, and with $k$ increased to $10^{-13} \mathrm{~m}^{2}$. 
reduces the displacement and strain of the cord, and thereby limits the magnitude of the cyclic swelling in the cord material, relative to what would occur in the absence of the pia. Pore pressure, as shown in the lower left-hand panel, is set by a combination of the pressures at the syrinx and SSS boundaries, the movements of pore fluid within the matrix, and the degree of distension of the porous matrix. The overall radial pressure gradient reflects instantaneously the Darcy flow of pore fluid through the matrix, but the pore pressure is rather constant spatially in the interior region where the seepage velocity is small, albeit its level varies basically with the syrinx pressure.

Since the whole cord and pia are porous, these phenomena are not confined to the tissues overlying the syrinx. Figure 9 shows corresponding traces for a radial cut through the solid cord and pia at $z=36.3$ $\mathrm{mm}$, about halfway between the cranial end of the syrinx and the cervical model boundary. The radial coordinate thus extends from zero to $5.85 \mathrm{~mm}$, with the cord/pia boundary at $5.65 \mathrm{~mm}$. In Fig. 8 , where $k=10^{-14} \mathrm{~m}^{2}$, the cyclic-swelling boundary layer thickness in the cord was of the order of 0.2 $\mathrm{mm}$. Here, the permeability has been increased to $10^{-13} \mathrm{~m}^{2}$, and the boundary layer thickness is correspondingly greater. The precise relation between the thickness of the cyclic swelling tissue boundary layer and the permeability will be explored below. The cord radial displacements (a consequence via the Poisson ratio of the time-varying axial strain of the cord at this location) here vary linearly with radial position, except close to the pia where the cyclic swelling becomes significant. Similarly, the pore fluid pressure variations are independent of radial position throughout most of the cord, since there is negligible seepage into the inner $4 \mathrm{~mm}$ of the cord radius. Despite the increased permeability, the magnitude of the fluid content changes is decreased relative to that in Fig. 8, because of the reduced radial displacements.

\section{Analysis}

We here sketch out an analytical solution to eqns. (1) to (4) for the simplified situation where only a transverse section through the syrinx and overlying cord at one axial position is considered; for more detail see [15]. For this purpose we consider only one poroelastic material layer, thus neglecting the pia; it can be reintroduced by applying appropriate boundary conditions at the interface between different tissues. The cord thus becomes a thick-walled annulus, of inner radius $r=a$ and outer radius $r$ $=b$, which is assumed to extend infinitely far without change in the axial direction. In the absence of body forces, and with inertial terms due to acceleration of pore fluid and poroelastic matrix assumed to be negligible, eqns. (1) and (2) reduce to

$$
\begin{gathered}
\widetilde{\nabla} \cdot \overline{\bar{\sigma}}=\tilde{0} \\
-\widetilde{\nabla} p_{p}-\frac{\mu}{k} \tilde{q}=\tilde{0}
\end{gathered}
$$

Together with eqns. (3) and (4), these are to be solved subject to the boundary conditions that, for the pore fluid, $\left.p_{\mathrm{p}}\right|_{r=a}=p_{\text {syrinx }}(t)$ and $\left.p_{\mathrm{p}}\right|_{r=b}=p_{\mathrm{SSS}}(t)$, and for the poroelastic matrix, $-\left.\tilde{n} \cdot(\overline{\bar{\sigma}} \cdot \tilde{n})\right|_{r=a}=$ $p_{\text {syrinx }}(t)$ and $-\left.\tilde{n} \cdot(\overline{\bar{\sigma}} \cdot \tilde{n})\right|_{r=b}=p_{\text {SSS }}(t)$. The equations are linear, and it can be assumed that all timevariables will execute simple harmonic motion, such that they can be represented by functions of the form $A^{*} e^{j \omega t}$, where $A^{*}$ is a complex number. This allows eqn. (4) to be written as

$$
j \omega \widetilde{\nabla} \cdot \tilde{u}+\widetilde{\nabla} \cdot \tilde{q}=0
$$

where the value $\alpha=1$ has been inserted; $\tilde{q}=\tilde{q}(r, z, t)$ in eqn. (7), but $\tilde{q}(r, z)$ here. Elimination of $\overline{\bar{\sigma}}$, $p_{p}$ and $\tilde{q}$ between eqns. (3), (6), (7) and (8) leads to a differential equation in the poroelastic matrix displacement $\tilde{u}$ 


$$
\frac{k}{j \omega \mu} \widetilde{\nabla} \cdot \widetilde{\nabla} \cdot(\lambda(\widetilde{\nabla} \cdot \tilde{u}) \overline{\bar{I}}+2 G \overline{\bar{e}})+\widetilde{\nabla} \cdot \tilde{u}=0
$$

where the Lamé constants $\lambda=\frac{E v}{(1+v)(1-2 v)}$ and $G=\frac{E}{2(1+v)}$. The solution to this equation which satisfies the boundary conditions (see [15] for more detail) is

$$
u(r)=\frac{C_{1}}{r}-A^{*} \frac{r}{2}+C_{2} J_{1}\left(j^{-1 / 2}\left(\frac{r}{\delta}\right)\right)+C_{3} K_{1}\left(j^{-3 / 2}\left(\frac{r}{\delta}\right)\right)
$$

where $A^{*}$ is now specifically the axial strain, $J_{1}()$ is a Bessel function of the first kind and first order, $K_{1}()$ is a modified Bessel function of the second kind and first order, $C_{1}, C_{2}$ and $C_{3}$ are constants taking values which satisfy the boundary conditions, and $\delta$, the cyclic-swelling boundary layer thickness, is given by

$$
\delta=\sqrt{\frac{k E(1-v)}{\omega \mu(1+v)(1-2 v)}}
$$

Thus the boundary layer is ten times thinner at $k=10^{-14} \mathrm{~m}^{2}$ than at $k=10^{-12} \mathrm{~m}^{2}$, and $\sqrt{250}$ or 15.8 times thicker in the stiff pia than in the soft cord material. The boundary layer thickness also depends inversely on the square root of the excitation frequency. Solutions for $p_{\mathrm{p}}$ and $\tilde{q}$ similar in form to eqn. (10) could be derived if needed. The fact that solutions of the poro-elastic equations develop boundary layers for small $k$ is well known [11], but to our knowledge their existence has not previously been noted in a neurological context.

The dependence of the cyclic-swelling boundary layer thickness $\delta$ on $k$ and $E$ can be seen in Figs. 8 and 9. In Fig. $8 \mathrm{~b}$, with $k=10^{-14} \mathrm{~m}^{2}, \delta$ can be seen to be of the order of $0.3 \mathrm{~mm}$ in the cord material overlying the syrinx. In the much stiffer pia, $\delta$ greatly exceeds the entire pia thickness of $0.2 \mathrm{~mm}$, and accordingly the spatial variations in seepage velocity indicate a boundary layer much thicker than the pia. In Fig. 9, with $k=10^{-13} \mathrm{~m}^{2}$, we expect $\delta$ in the cord to increase by a factor of $\sqrt{10}$, to around 0.95 $\mathrm{mm}$, as is indeed shown by Fig. $9 \mathrm{~b}$. In the pia, $\delta$ exceeds the pia thickness still more than at $k=10^{-14}$ $\mathrm{m}^{2}$, and again the seepage velocity variations indicate a boundary layer thickness much greater than the pia width.

\section{Discussion}

We have implemented a poroelastic version of the axisymmetric FSI model of the cord, pia, and dura with SSS stenosis and co-located syrinx of Bertram [2]. We find that the time-varying cord displacements in response to sine waves of pressure applied at the cervical end of the SSS are largely unchanged from those seen in the impermeable model. The peak-to-peak excursion of the time-varying pressure in the SSS caudal to the stenosis and of that in the syrinx is slightly increased relative to the impermeable situation. As found earlier [2;23], the time-varying stenotic gap acts to cause mean pressure in the caudal SSS to be elevated relative to that cranial to the stenosis ${ }^{6}$. This remains so, but

\footnotetext{
${ }^{6}$ The 'leaky valve' effect of the stenosis is a global nonlinearity which does not depend on either of the two local nonlinearities which were noted in the context of Fig. 3 to cause depression of the mean pressure in the stenosis gap. This can be seen by supposing that gap width and gap flow both vary in phase with applied pressure. The average gap width for caudally directed flow is then greater that for cranially directed flow, and elevated mean pressure in the caudal SSS results. Neither a Bernoulli effect nor viscous resistance increasing nonlinearly with gap narrowness are necessary to this argument.
} 
the elevation is slightly less in the permeable model. The pulsatile flow through the stenosis is now shunted by a parallel flow via the syrinx and the porous media overlying it, but with realistically low values of the cord/pia permeability, this shunt flow is small relative to the flow through the stenosis itself. In fact very little of the radial pulsatile flow which passes through the SSS/pia interface makes it as far as the syrinx; most is absorbed in the overlying porous media, particularly in the soft cord material, causing a periodic swelling of these tissues and concomitant increase in their fluid content. However there is also now a weak steady streaming flow, driven by the leaky valve of the flexible stenosis around a circuit craniocaudally past the stenosis, into the caudal half of the syrinx via the porous cord and pia, caudocranially along the syrinx, and back out into the SSS via the porous media overlying the cranial half of the syrinx.

Steady streaming occurs when the SSS stenosis is located halfway along the adjacent syrinx. However, more significant changes in syrinx volume occur when the stenosis and syrinx are axially offset. If the syrinx is positioned $30 \mathrm{~mm}$ more cranially, it loses volume relative to its starting situation. If it is positioned an equal distance more caudally, it gains volume. In our poroelastic model, such volume changes are inevitably bounded; they come to an end once a cycle-average equilibrium of the syrinx and SSS pressures (Fig. 3), the circular streaming flow (Fig. 5) and the strain and fluid content of all the porous structures has been reached in response to the excitation. The time-scale of this evolution may extend to more than 100 cycles of excitation, but is very short compared to the time-scale of syrinx growth in vivo. In the biological situation, such an endpoint is likely to be impermanent, because of subsequent tissue remodelling. Over long time periods, remodelling could potentially amplify the effect of the fluid-dynamic processes giving rise to volume change here. The changes in time-average volume we compute are small, but they do suggest that a syrinx is more likely to grow by this mechanism just caudal to a site of SSS obstruction than cranially. The clinical literature is ambivalent on this point. Brodbelt \& Stoodley [6] reported that "post-traumatic syringes are usually juxtaposed to the injury site and extend rostral in $81 \%$, caudal in $4 \%$, and in both directions in $15 \%$ of cases." Klekamp [21] qualified this, stating that "arachnoid scarring in the cervical area will generally cause a syrinx below the spinal level of arachnoid scarring, whereas thoracic arachnoid scars lead to an upward expansion of a syrinx." For intramedullary tumours, Samii and Klekamp [28] found that associated syrinxes occurred above (49\%), below (11\%) and both above and below (40\%) the level of the tumour. On the other hand, syrinxes associated with Chiari I malformation typically occur caudal to the site of cerebellar tonsillar herniation; thus quoting Oldfield et al. [25], "In all [seven] patients the cerebellar tonsils occluded the subarachnoid space at the level of the foramen magnum. Syringomyelia extended from the cervical to the lower thoracic segment of the spinal cord."

As in the impermeable model, the mean pressure in the syrinx is elevated relative to that in the SSS cranial to the stenosis. This may explain the instances when pressure measurements in animals or patients have found higher pressure in the syrinx than in the neighbouring SSS $[10 ; 12 ; 14 ; 24]$.

It was previously hypothesised $[2 ; 13]$ that elevated mean pressure in the caudal SSS relative to that in the syrinx might provide a means whereby fluid could seep from the SSS to the syrinx, i.e. a means of filling it; however, on the basis of the impermeable model the hypothesis could not be tested. The results here from the poroelastic model confirm that the combination of permeable media and the nonlinearity provided by the 'leaky valve' of the SSS stenosis does provide such a mechanism, albeit quantitatively a very weak one, as evidenced by the admittedly tiny changes in mean syrinx volume.

In reality gap width does vary approximately in phase with applied pressure, while the flow through the gap, although locally dominated by viscous effects, lags behind the applied pressure because of the inertial dominance of SSS flow in general, but the lag is not sufficient to invalidate the simple argument presented. 
The mechanism is highly sensitive to the placement of the syrinx relative to the stenosis, such that the filling tendency if the majority of the syrinx is situated caudal to the stenosis turns to an emptying one if it is predominantly situated cranially. This finding of course relies on the axial uniformity of permeability in our model; one can envisage how axially non-uniform permeability of the overlying tissues might move the filling/emptying balance point. Whether the mechanism could over extended time cause major changes in syrinx volume remains unknown. Nevertheless, we have demonstrated that the mechanism works in principle, in the process providing an alternative to the mechanism of syrinx filling via perivascular spaces proposed by Bilston et al. [5].

We have also demonstrated that fluid in the interstitium of the spinal cord can exchange with the neighbouring CSF, simply as a result of the porosity of the cord and its covering pia mater, combined with the continuous slight oscillation of SSS pressure as a result of cranial arterial pulsation. More significant transient perturbations in SSS pressure can arise from coughing [29;32]. The exchange process is confined to a thin boundary layer just under the pia, but it is nevertheless sufficient to substantiate through numerical modelling that there is no real dividing line between interstitial fluid (ISF) and CSF (already understood via physiological experimentation-see [20] and references therein). The process is dependent on the extent of local pulsatile tissue strain. Thus it is largest in the thinned cord overlying the syrinx, and much less (but still non-zero) in the solid cord away from the syrinx, where the axial and radial components of pulsatile strain are smaller. Similarly, extrapolating beyond our spinal model, we can expect that it would be even less at the pial surface of the brain. Nevertheless, it may have a bearing on current controversies generated by tracer studies. Thus Nedergard et al. [17-18] have proposed that CSF moves into the interstitium along periarterial channels and back out again via perivenous ones, in the process clearing waste products of brain metabolism. Conversely, Weller et al. [8] have proposed that solutes drain from the brain to the CSF along periarterial basement membranes. A common thread is that tracer injected at one site and later found elsewhere is used as evidence for flow, albeit in the absence of a fluid-dynamic mechanism (a pressure gradient) for such flow. Both sets of findings can be explained, at least in part, by means of the ISF/CSF mixing ${ }^{7}$ in the cyclic-swelling boundary layer of the parenchyma that we have demonstrated.

Finally, the many limitations of our study must be acknowledged. The spinal cord and subarachnoid space have been modelled in isolation from the brain, and in a highly idealised axisymmetric geometry. Whereas in reality the cord exists to fan out nerves along its length, here it was reduced to a tapering poroelastic rod, and likewise the trabeculae and denticulate ligaments that inhabit the SSS were omitted. The cord and the pia had the same uniform and isotropic permeability, although it is known that cord permeability is anisotropic [27]. The periodic impulsion of CSF from the cerebral subarachnoid space to the SSS was reduced to a sine wave of pressure applied at the cervical end of the model, where axial displacement of the cord and dura was prevented. Despite a substantial syrinx, the outer cord diameter was maintained unchanged locally. The many and varied situations giving rise to SSS stenosis were reduced to an elastic protrusion from the dura. Although parameter values were chosen to emulate reality as far as possible, these and other simplifications mean that close quantitative agreement between the predictions of the model and real syringomyelia with SSS stenosis should not be looked for. The model exists primarily as a physics test-bed for hypotheses about possible hydrodynamic mechanisms to do with syringogenesis. As such its utility lies in distinguishing between what is mechanically feasible and what is not. We have shown that the nonlinearity of a slightly flexible stenosis of the SSS, in conjunction with the porosity of the neural parenchyma, provides a

\footnotetext{
${ }^{7}$ Mixing actually requires a further step beyond just the oscillatory flow of pore fluid across the pia/SSS boundary: the diffusion of solutes down their concentration gradient. In combination, in the process called Taylor dispersion, solutes can move continuously in one direction as the result of a purely oscillatory flow.
} 
possible mechanism for syrinx enlargement, albeit a weak one. We also demonstrate that ISF and CSF can exchange and mix with each other in a thin tissue boundary layer just inside the pia, and this has implications that go beyond syringomyelia.

\section{Acknowledgements}

We thank the Chiari and Syringmyelia Foundation for the grant to CDB which funded the commencement of this work. Matthew J. Russell (student supervised by MH) contributed to the implementation of the poro-elastic FSI capabilities in oomph-lib.

\section{References}

[1] Bertram, C.D. (2009), A numerical investigation of waves propagating in the spinal cord and subarachnoid space in the presence of a syrinx. Journal of Fluids and Structures 25(7), 11891205. DOI: 10.1016/j.jfluidstructs.2009.06.008

[2] Bertram, C.D. (2010), Evaluation by fluid/structure-interaction spinal-cord simulation of the effects of subarachnoid-space stenosis on an adjacent syrinx. ASME Journal of Biomechanical Engineering 132(6), 061009-1-15.

[3] Bertram, C.D. (2012), Benchmarking of fluid/structure interaction models of wave propagation (poster), in ECI Conference on Computational Fluid Dynamics in Medicine and Biology, and 7th International Biofluid Mechanics Symposium, Ein Bokek, Dead Sea, Israel.

[4] Bertram, C.D., Brodbelt, A.R., Stoodley, M.A. (2005), The origins of syringomyelia: numerical models of fluid/structure interactions in the spinal cord. ASME Journal of Biomechanical Engineering 127(7), 1099-1109.

[5] Bilston, L.E., Stoodley, M.A., Fletcher, D.F. (2010), The influence of the relative timing of arterial and subarachnoid space pulse waves on spinal perivascular cerebrospinal fluid flow as a possible factor in syrinx developments. Journal of Neurosurgery 112(4), 808-813.

[6] Brodbelt, A.R., Stoodley, M.A. (2003), Post-traumatic syringomyelia: a review. Journal of Clinical Neuroscience 10(4), 401-408.

[7] Brugières, P., Idy-Peretti, I., Iffenecker, C., Parker, F., Jolivet, O., Hurth, M., Gaston, A., Bittoun, J. (2000), CSF flow measurement in syringomyelia. American Journal of Neuroradiology 21(10), 1785-1792.

[8] Carare, R.O., Bernardes-Silva, M., Newman, T.A., Page, A.M., Nicoll, J.A.R., Perry, V.H., Weller, R.O. (2008), Solutes, but not cells, drain from the brain parenchyma along basement membranes of capillaries and arteries: significance for cerebral amyloid angiopathy and neuroimmunology. Neuropathology and Applied Neurobiology 34(2), 131-144. DOI: 10.1111/j.1365-2990.2007.00926.x

[9] Cloyd, M.W., Low, F.N. (1974), Scanning electron microscopy of the subarachnoid space in the dog. I. Spinal cord levels. Journal of Comparative Neurology 153(4), 325-367.

[10] Davis, C.H.G., Symon, L. (1989), Mechanisms and treatment in post-traumatic syringomyelia. British Journal of Neurosurgery 3, 669-674.

[11] Detournay, E., Cheng, A.H.-D. (1993), Fundamentals of poroelasticity, in: Comprehensive Rock Engineering: Principles, Practice and Projects, Vol. II, Analysis and Design Method, C. Fairhurst, (ed.), Pergamon Press, pp. 113-171.

[12] Ellertsson, A.B., Greitz, T. (1970), The distending force in the production of communicating syringomyelia. Lancet 295(7658), 1234. 
[13] Elliott, N.S.J., Bertram, C.D., Martin, B.A., Brodbelt, A. (2013), Syringomyelia: a review of the biomechanics. Journal of Fluids and Structures 40, 1-24. DOI:

10.1016/j.jfluidstructs.2013.01.010

[14] Hall, P., Turner, M., Aichinger, S., Bendick, P., Campbell, R. (1980), Experimental syringomyelia: the relationship between intraventricular and intrasyrinx pressures. Journal of Neurosurgery 52(6), 812-817.

[15] Heil, M., Bertram, C.D. A poroelastic fluid-structure interaction model of syringomyelia. Journal of Fluid Mechanics (submitted)

[16] Heil, M., Hazel, A.L. (2006), oomph-lib - An Object-Oriented Multi-Physics Finite-Element Library, in: Fluid-Structure Interaction, M. Schafer, H.-J. Bungartz, (eds.), Springer (Lecture Notes on Computational Science and Engineering), pp. 19-49.

[17] Iliff, J.J., Lee, H., Yu, M., Feng, T., Logan, J., Nedergaard, M., Benveniste, H. (2013), Brainwide pathway for waste clearance captured by contrast-enhanced MRI. Journal of Clinical Investigation 123(3), 1299-1309. DOI: 10.1172/JCI67677

[18] Iliff, J.J., Wang, M., Zeppenfeld, D.M., Venkataraman, A., Plog, B.A., Liao, Y., Deane, R., Nedergaard, M. (2013), Cerebral arterial pulsation drives paravascular CSF-interstitial fluid exchange in the murine brain. The Journal of Neuroscience 33(46), 18190-18199. DOI: 10.1523/JNEUROSCI.1592-13.2013

[19] Jäger, W., Mikelić, A. (2000), On the interface boundary condition of Beavers, Joseph, and Saffman. SIAM Journal of Applied Mathematics 60(4), 1111-1127.

[20] Klekamp, J. (2002), The pathophysiology of syringomyelia - historical overview and current concept. Acta Neurochirurgica 144, 649-664.

[21] Klekamp, J. (2009), Syringomyelia, in: Practical Handbook of Neurosurgery, pp. 145--161.

[22] Levine, D.N. (2004), The pathogenesis of syringomyelia associated with lesions at the foramen magnum: a critical review of existing theories and proposal of a new hypothesis. Journal of the Neurological Sciences 220, 3-21.

[23] Martin, B.A., Labuda, R., Royston, T.J., Oshinski, J.N., Iskandar, B., Loth, F. (2010), Spinal canal pressure measurements in an in vitro spinal stenosis model: implications on syringomyelia theories. ASME Journal of Biomechanical Engineering 132, 111007-1-11.

[24] Milhorat, T.H., Capocelli, A.L., Kotzen, R.M., Bolognese, P., Heger, I.M., Cottrell, J.E. (1997), Intramedullary pressure in syringomyelia: clinical and pathophysiological correlates of syrinx distension. Neurosurgery 41(5), 1102-1110.

[25] Oldfield, E.H., Muraszko, K., Shawker, T.H., Patronas, N.J. (1994), Pathophysiology of syringomyelia associated with Chiari I malformation of the cerebellar tonsils. Journal of Neurosurgery 80, 3-15.

[26] Ozawa, H., Matsumoto, T., Ohashi, T., Sato, M., Kokubun, S. (2004), Mechanical properties and function of the spinal pia mater. Journal of Neurosurgery (Spine) 1, 122-127.

[27] Rossi, C., Boss, A., Steidle, G., Martirosian, P., Klose, U., Capuani, S., Maraviglia, B., Claussen, C.D., Schick, F. (2008), Water diffusion anisotropy in white and gray matter of the human spinal cord. Journal of Magnetic Resonance Imaging 27(3), 476-482. DOI: 10.1002/jmri.21252

[28] Samii, M., Klekamp, J. (1994), Surgical results of 100 intramedullary tumors in relation to accompanying syringomyelia. Neurosurgery 35(5), 865--873.

[29] Sansur, C.A., Heiss, J.D., DeVroom, H.L., Eskioglu, E., Ennis, R., Oldfield, E.H. (2003), Pathophysiology of headache associated with cough in patients with Chiari I malformation. Journal of Neurosurgery 98(3), 453-458.

[30] Simon, B.R. (1992), Multiphase poroelastic finite element models for soft tissue structures. Applied Mechanics Reviews 45, 191-218. 
[31] Smillie, A., Sobey, I., Molnar, Z. (2005), A hydroelastic model of hydrocephalus. Journal of Fluid Mechanics 539, 417-443.

[32] Williams, B. (1976), Cerebrospinal fluid pressure changes in response to coughing. Brain 99, 331-346.

[33] Williams, B. (1980), On the pathogenesis of syringomyelia: a review. Journal of the Royal Society of Medicine 73, 798-806. 


\section{CAPTIONS}

Table 1 Mean syrinx volume changes with a shift in syrinx position and with $k$. Whole cord and pia porous, with $\alpha=1$. Monoexponential curves fitted to running cycle-averages at $t \geq 1 \mathrm{~s}$ were extrapolated beyond $\sim 5 \mathrm{~s}$ to find the final $\Delta$ (volume).

Figure 1 The model geometry, at 1:1 scale on the left and radially exaggerated $20 \times$ on the right. Aqueous fluid in blue. Three solids having different stiffnesses are shown in differing colours; solid colour denotes an impermeable solid, blue-chequered colour denotes a porous medium.

Figure 2 The deflection (a, b) of the FSI boundaries of the model, magnified $5 \times$, and the pressure along the inner boundary of the SSS (solid line) and (broken line) on the syrinx centre-line $(c, d)$, both shown at the peak (a, c) and the trough (b, d) of the forcing cycle. The permeable model outline (red) almost entirely overlaps that of the impermeable model (blue). The real change in gap width is much smaller than depicted in the exaggerated views $(a, b)$ shown here.

Figure 3 Profiles of cycle-average pressure vs. axial position at the inner (solid lines) and outer (dotted) edges of the SSS, and at the syrinx centre-line (dashed). Only the axial region $70<$ $z<230 \mathrm{~mm}$, which spans the syrinx, is shown. Results are shown for the non-porous cord and pia (blue), with cord and pia porous only over the syrinx (green, "cov-por"), and with the whole cord and pia porous (red, "all-por").

Figure 4 Upper panels compare the flow-rate through the stenosis gap (blue, "thru gap") with that arriving in the caudal SSS via the syrinx and overlying porous media (green, "crossing iSSS caudally"-iSSS denotes the inner boundary of the SSS). Lower panels compare the same flow-rate entering the caudal SSS (green, "thru SSS") with that leaving the caudal half of the syrinx (red). Start-up transients are visible over the first three cycles $(1.2 \mathrm{~s})$.

Figure 5 Steady streaming flow.

Figure 6 (a, c) Instantaneous changes in syrinx volume at $k=10^{-13} \mathrm{~m}^{2}$, with $(\mathrm{b}, \mathrm{d})$ the corresponding running averages over a cycle. Panels (a) and (b) compare (red) the result when the whole cord and pia are fully poroelastic $(\alpha=1)$ to (green) that when the cord and pia are poroelastic only over the syrinx, and (blue) Darcy flow only occurs over the syrinx. Panels (c) and (d) compare (red) the same whole-cord-poroelastic result with that when the syrinx is displaced axially ("shift") by (beige) $+30 \mathrm{~mm}$ or (black) $-30 \mathrm{~mm}$ relative to the stenosis.

Monoexponentials are fitted to the curves in (d) and extrapolated to find the asymptotic values (dashed lines).

Figure 7 The solids outline and the fluid pressure at four equi-spaced times during an excitation cycle, with the syrinx displaced $30 \mathrm{~mm}$ caudally. Colour key shows pressure in Pa. Radial scale $10 \times$ axial scale, displacements exaggerated $20 \times$.

Figure 8 Instantaneous profiles of (a) radial displacement, (b) seepage velocity, (c) pore pressure, and (d) the divergence of seepage velocity, for eight equi-spaced times through the cycle, vs. 
radial position within a cut through the cord and pia overlying the syrinx as indicated in the sketch on the left. In each panel the syrinx is to the left, and the SSS to the right.

Figure 9 Traces as in Fig. 8, but for a radial cut through the cord cranial to the syrinx, and with $k$ increased to $10^{-13} \mathrm{~m}^{2}$. 